\title{
Genome-wide insights into lipid levels
}

As for many complex traits, genomewide association (GWA) studies are revolutionizing the dissection of the genetic determinants of blood lipid levels, a known risk factor for cardiovascular disease. Three recent reports highlight how differences in study design can provide complementary insights and identify novel risk loci that could improve our understanding of lipoprotein metabolism.

The large numbers of individuals studied gave the new research increased power relative to previous studies, and their use of population cohorts reduced some of the potential biases of case-control studies. These studies both confirm risk loci identified in previous analyses and uncover several new candidate genetic determinants for lipid levels.

Aulchenko and colleagues identified genetic variants associated with a range of lipid traits, including total cholesterol (TC) and low-density lipoprotein (LDL) cholesterol, in 16 diverse European population cohorts - a total of 24,000 individuals. The effect sizes of loci with significant associations were largely the same across populations. However, for three loci there was a significant difference between males and females, providing genetic support for previous clinical observations of gender differences in lipid levels. They also calculated genetic risk scores for the oldest population cohort, and found the genetic risk profiles predicted clinically relevant outcomes, such as the thickness of blood-vessel walls.

The Framingham Heart Study cohort, in which TC was first established as a risk factor for cardiovascular disease, was included in a study of $\sim 40,000$ individuals by Kathiresan and colleagues. For several of the 30 loci that they linked to lipid levels these authors showed that multiple genetic variants - both common and rare - contribute to lipid-level variation; modelling multiple variants per locus increased the proportion of variance in lipid levels explained by these loci. They also showed that lipid-associated SNPs could influence

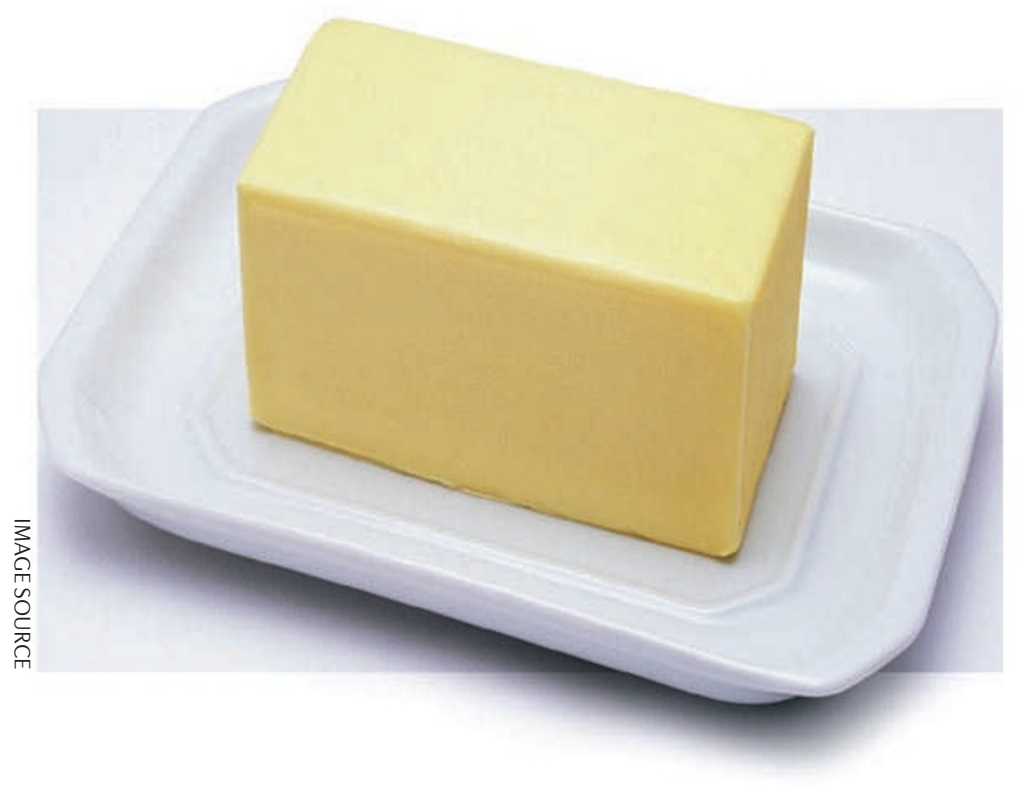

mRNA levels in the liver. Such methods highlight the biological significance of genetic variants.

In a third study, Sabatti and colleagues looked for associations with various metabolic traits, including lipid levels, in a very different population cohort: the Northern Finland Birth Cohort 1966. This cohort is relatively homogeneous in terms of genetic background and environmental exposures. Among their findings, they showed an association of LDL levels with an infrequent SNP in the androgen receptor gene. The authors suggest that studies of genetic isolates might provide improved power for the notoriously difficult task of detecting association to rare variants, as the frequency of such variants can vary substantially between populations.

Although these studies add to the list of loci that are reliably associated with lipid levels, these loci explain only a proportion of the heritable component of this complex trait. However, the range of approaches these studies use helps to build up a picture of the biological basis of lipid levels, and could offer clinicians new avenues for reducing heart disease risk.

Mary Muers

ORIGINAL RESEARCH PAPERS Aulchenko, Y.S et al. Genome-wide association study in 16 European cohorts: major loci influencing lipid levels and coronary heart disease risk. Nature Genet. 7 Dec 2008 (doi:10.1038/ng.269)| Kathiresan, S. et al. Common DNA sequence variants at thirty genetic loci contribute to polygenic dyslipidemia. Nature Genet. 7 Dec 2008 (doi:10.1038/ng.277)| Sabatti, C. et al. Genome-wide association analysis of metabolic traits in a birth cohort from a founder population. Nature Genet. 7 Dec 2008 (doi: 10.1038/ng.271) FURTHER READING Lusis, A. J. et al. Metabolic syndrome: from epidemiology to systems biology. Nature Rev. Genet. 9, 819-830 (2008) 